\title{
Coptos (2020)
}

\section{Laure Pantalacci et Cédric Gobeil}

\section{CpenEdition \\ Journals}

Édition électronique

URL : https://journals.openedition.org/baefe/2780

DOI : $10.4000 /$ baefe. 2780

ISSN : 2732-687X

Éditeur

ResEFE

\section{Référence électronique}

Laure Pantalacci, Cédric Gobeil, « Coptos (2020) » [notice archéologique], Bulletin archéologique des

Écoles françaises à l'étranger [En ligne], Égypte, mis en ligne le 30 mai 2021, consulté le 27 juillet 2021 URL : http://journals.openedition.org/baefe/2780 ; DOI : https://doi.org/10.4000/baefe.2780

Ce document a été généré automatiquement le 27 juillet 2021.

\section{c) (i) (3)}

Le Bulletin archéologique des Écoles françaises à l'étranger est mise à disposition selon les termes de la Licence Creative Commons Attribution - Pas d'Utilisation Commerciale - Pas de Modification 4.0

International. 


\title{
Coptos (2020)
}

\author{
Laure Pantalacci et Cédric Gobeil
}

\section{NOTE DE L'AUTEUR}

Année de la campagne : 2020 ( $1^{\text {er }}$ février - 5 mars)

Autorité nationale présente : Le ministère du Tourisme et des Antiquités (MoTA) était représenté par Marwa Gamal El-Din Zaki et le Département des Projets de Qena-Qift par Mohammed Ahmed Hussein

Numéro et intitulé de l'opération de terrain : 17143 - Coptos, la ville et ses monuments. Fouilles et valorisation du site

Composition de l'équipe de terrain : L'équipe était composée de Laure Pantalacci (chef de mission, égyptologue, université Lumière Lyon 2, UMR 5189), Cédric Gobeil (égyptologue archéologue, Museo Egizio, Turin, UMR 5189), Vincent Chollier (ATER, université Lumière Lyon 2), Vanessa Desclaux (égyptologue épigraphiste, BnF, UMR 5189), Anne-Charlotte Ybard (archéologue dessinatrice, SAVL), Samuel Louvion (architecte, DPLG), Clemens Thienpont (tailleur de pierre indépendant), Beatrice de Faveri (MA, Università di Bologna), Valentine Marlot (doctorante, université Lumière Lyon 2), Ebeid Mahmoud Hamid (restaurateur, Ifao) et Ihab Mohammed Ibrahim (photographe, Ifao).

Partenariats institutionnels :

- MoTA

- CNRS/université Lumière Lyon 2 - UMR 5189 Histoire et sources des mondes antiques HiSoMA

- Museo Egizio, Turin

- UMR 7298 LA3M

Organismes financeurs :

- CNRS/université Lumière Lyon 2 - UMR 5189

- Museo Egizio, Turin

- Fonds Khéops pour l'archéologie 
Donnée scientifiques produites :

https://coptos.mom.fr/

\section{Problématique et bilan des principaux résultats}

- Étude de l'évolution architecturale et urbaine des centres religieux de la ville, de l'époque pharaonique à l'époque romaine impériale.

- Découverte et fouille d'un mammisi construit par Ptolémée IV.

- Identification d'un temple d'Isis et Harpocrate décoré par Ptolémée IX.

- Mise en évidence des réaménagements successifs des ensembles cultuels durant l'époque gréco-romaine.

- Anastylose de petits monuments d'époque romaine.

\section{Travaux réalisés en 2020}

\subsection{Mission}

La mission Ifao/université Lumière Lyon 2 s'est déroulée du $1^{\text {er }}$ février au 5 mars 2020. Les travaux de cette saison se sont concentrés de nouveau sur les deux secteurs de la colonnade nord et de la chapelle orientale d'Isis, avec une brève intervention sur le mammisi (fig. 1).

Fig. 1. Secteurs des travaux réalisés en 2020 (D. Laisney, G. Soukiassian, C. Gobeil).

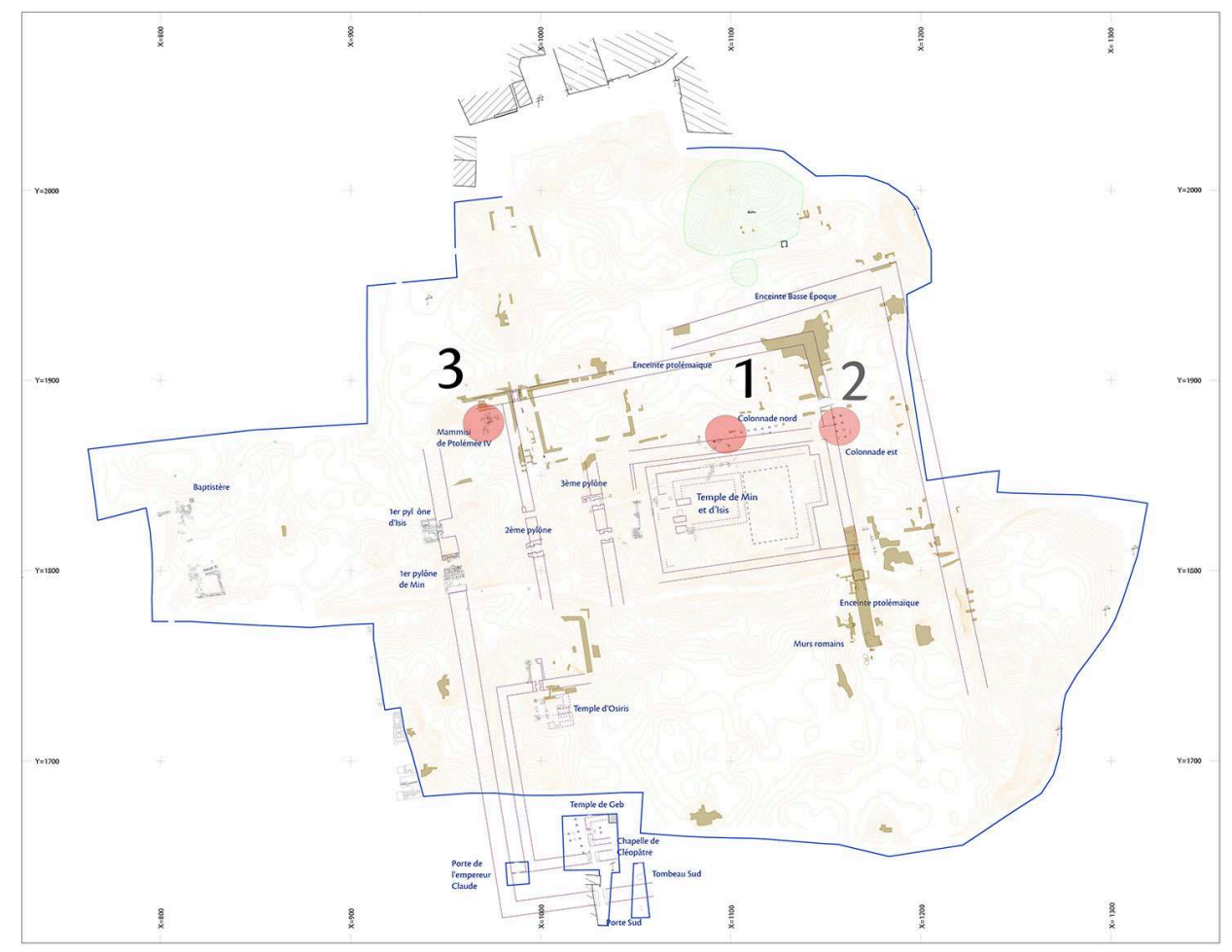

(C) Ifao. 17143_2020_NDMCN_001 


\subsubsection{La colonnade nord et le mur nord du temple}

Cédric Gobeil, Valentine Marlot, Beatrice de Faveri, Samuel Louvion, Vanessa Desclaux, Vincent Chollier

2 L'examen archéologique de la colonnade romaine située au nord du temple de Min et d'Isis a de nouveau été poursuivi cette année. L'essentiel des travaux a consisté à retirer le kôm de déblais - probablement créé lors des fouilles de Petrie en 1894 et étendu par les sebbakhin à sa suite - qui recouvrait jusqu'alors le mur de briques crues hellénistique. Les terres meubles du monticule de déblais sont mêlées de tessons céramiques, en majorité d'époque romaine et byzantine, et de fragments ou éclats de calcaire et de grès d'assez petit module, témoins de la destruction du temple.

Durant cette campagne, l'objectif était d'étendre vers l'ouest le nettoyage de la double rangée de colonnes et du rang de piliers entamé précédemment. Un premier secteur de $25 \times 15 \mathrm{~m}$ a été ouvert dans la continuité du secteur déjà nettoyé (fig. 2). La tâche principale a consisté à retirer l'épaisse couche de déblais (US CN-1) qui recouvrait l'ensemble du secteur. Durant cette opération, plusieurs résultats ont été obtenus.

Fig. 2. La colonnade nord, vue nord-sud (C. Gobeil).

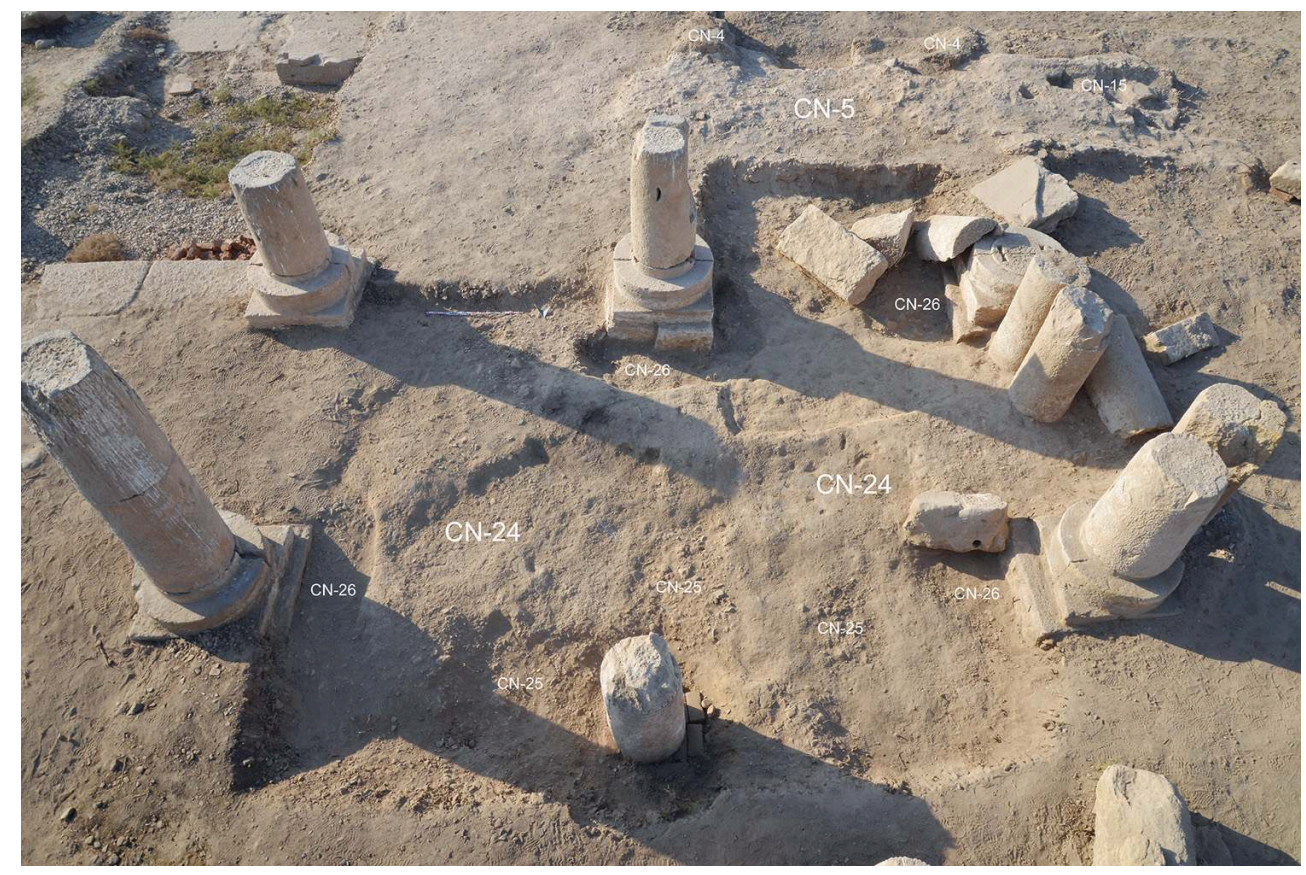

(C) Mission Coptos. 17143_2020_NDMPF_001

Tout d'abord, la limite ouest du mur de briques crues $\mathrm{CN}-4$ a été identifiée, contre laquelle vient s'appuyer une épaisse couche de mortier blanc induré (US CN-5, ép. $50 \mathrm{~cm}$ ) déjà exposée la saison précédente. Le mortier de cette couche est mélangé à de très nombreux éclats de calcaire ainsi qu'à quelques plus gros fragments de pierre calcaire, laissant alors penser qu'une activité de débitage a eu lieu là après que la colonnade nord eut cessé d'être utilisée comme voie de circulation. Le niveau irrégulier de la couche $\mathrm{CN}-5$, dans laquelle de petites fosses ( $\mathrm{CN}-15)$ ont été aménagées, laisse penser qu'elle a également pu servir de surface de travail une fois les travaux de débitage terminés. 
Des éléments architecturaux de grand module provenant du démontage du temple ont été mis au jour dans la colonnade, parmi lesquels un bloc de grès formant un angle et décoré sur trois de ses côtés (inv. 2820), montrant un pharaon faisant l'offrande de la campagne, et neuf colonnes subsistant d'un long texte. De nouveaux éléments de la colonnade elle-même ont aussi été découverts. Parmi ceux qui méritent une attention particulière se trouvent un chapiteau composite polychrome (inv. CN33, visible à gauche sur la fig. 3), et la partie supérieure d'un fût de colonne d'un type jusqu'ici inédit dans le secteur fouillé. Ce fût reposait sur un sol de belle qualité fait de briques crues alignées (US CN-24) recouvertes d'une très mince couche de mortier gris clair induré et lisse. Ce sol est coupé par quelques fosses circulaires peu profondes (US CN-25) et par des tranchées de fondation de forme plus ou moins régulière (US CN-26) aménagées lors de l'installation de chacune des bases de colonne. Il est donc intéressant de noter que ce sol $\mathrm{CN}-24$ précède l'implantation de la colonnade nord ; du reste, cette antériorité n'exclut pas qu'il ait pu faire partie intégrante du même projet. L'horizontalité relativement homogène du sol $\mathrm{CN}-24$ laisse penser qu'il a pu, au moins temporairement, être recouvert de dalles de pierre similaires à celles qui sont toujours en place aujourd'hui à l'est du secteur fouillé.

Dans l'axe de la colonnade, à 5 mètres à l'ouest des dernières colonnes encore en élévation, des murs de briques crues (US CN-32 à 37, fig. 3), conservés sur une hauteur de seulement quelques assises, ont été découverts immédiatement sous la surface $\mathrm{CN}-1$. Le niveau de fondation de ces structures $(75,80 \mathrm{~m})$ et les petites dimensions de leurs briques $(24 \times 12 \times 6 \mathrm{~cm})$ indiqueraient qu'il s'agit de constructions byzantines. Ces murs ayant été découverts durant la dernière semaine de fouilles, ils seront étudiés plus en détail lors de la prochaine campagne.

Fig. 3. Murs byzantins à l'ouest des colonnes conservées, vue nord-est/sud-ouest ; à gauche, le chapiteau composite CN-33 (C. Gobeil).

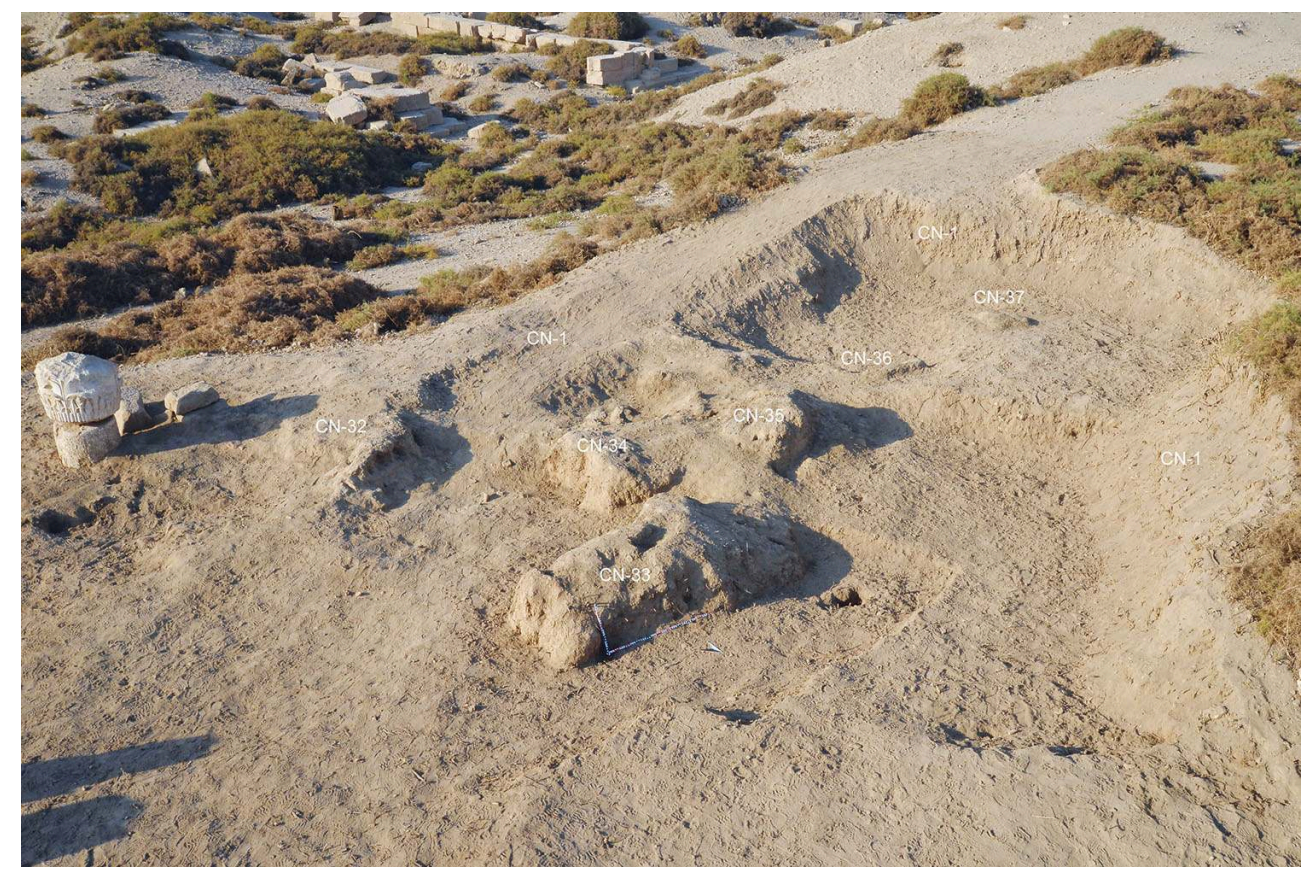

(c) Mission Coptos. 17143_2020_NDMPF_002 
7 Une seconde opération a été menée afin de vérifier la nature du seul pan de mur de briques crues est-ouest (US CN-11) resté en élévation immédiatement au nord de la colonnade. Par hypothèse, ce pan de mur était compris comme étant le dernier élément encore visible du mur-écran qui devait fermer la colonnade au nord (fig. 4). Les travaux ont permis de constater que ce mur était en effet structurellement connecté à la rangée de bases de piliers en calcaire implantée parallèlement à la double rangée de colonnes. Les deux faces du mur $\mathrm{CN}-11$ ont été identifiées, permettant ainsi de reconnaître l'épaisseur d'origine du mur qui était de 2,11 mètres, soit quatre coudées. L'une des principales caractéristiques de ce mur est son appareil de briques crues alternant deux couleurs différentes, à la façon d'un damier: une brique beige-rosé est systématiquement posée à côté d'une brique marron foncé, et ainsi de suite (fig. 5). Dans la mesure où le mur, à l'origine, devait être recouvert d'un enduit, cette alternance de couleur ne devait pas avoir de but esthétique. L'explication est plutôt à chercher du côté de la résistance des différents matériaux employés : tandis que les briques beige-rosé sont sableuses, les briques marron foncé sont quant à elles argileuses. Par conséquent, ce mode de construction, dont nous n'avons pas pour l'instant trouvé de parallèle, devait assurer au mur à la fois une certaine élasticité et une grande robustesse. La recherche du niveau de fondation du mur $\mathrm{CN}-11$ a permis de mettre au jour un mur de briques crues plus ancien (US CN-23) conservé sous sa fondation et séparé de $\mathrm{CN}-11$ par un mince remblai de terre marron assez meuble mélangée à de petits tessons (US $\mathrm{CN}-22$ ), ayant servi de nivellement au mur $\mathrm{CN}-11$. Une fouille plus approfondie du secteur devrait permettre, l'année prochaine, de comprendre la nature du mur $\mathrm{CN}-23$.

Tous les fragments décorés trouvés lors de ce nettoyage ont été enregistrés dans la base de données, photographiés et dessinés par V. Desclaux et V. Chollier.

Fig. 4. Le mur nord de la colonnade $\mathrm{CN}-11$, vue sud-ouest/nord-est (C. Gobeil).

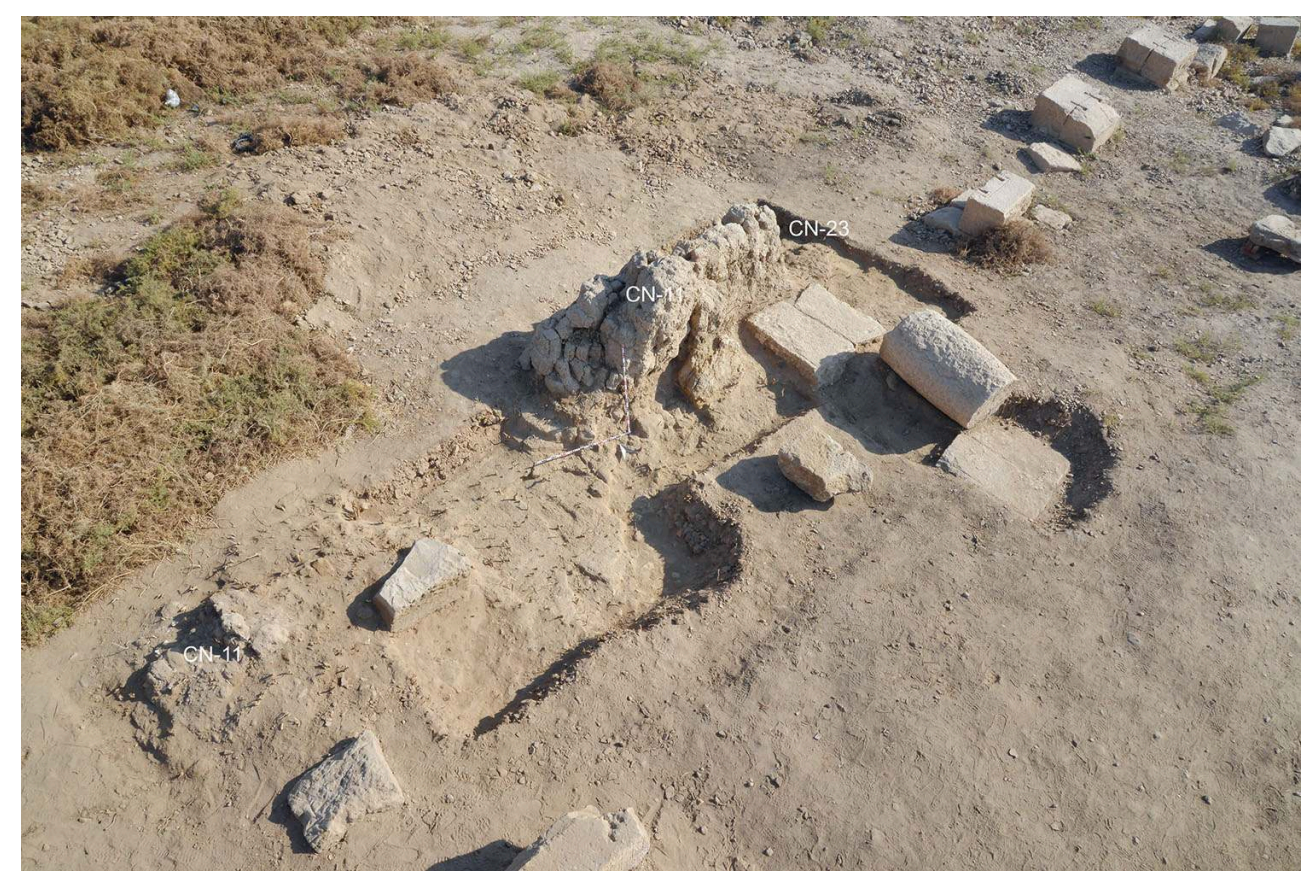

(c) Mission Coptos. 17143_2020_NDMPF_003 
Fig. 5. Détail du mur $\mathrm{CN}-11$, alternance en damier de briques beiges et marron (C. Gobeil).

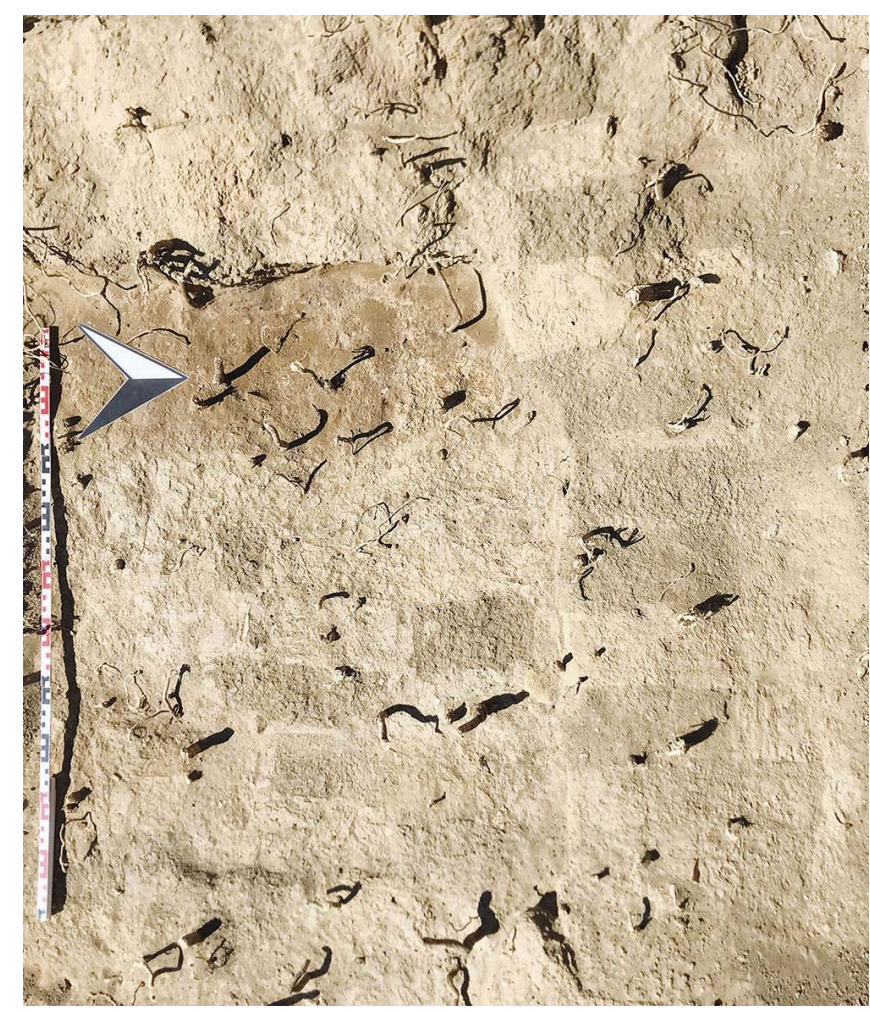

(c) Mission Coptos. 17143_2020_NDMPF_004

\subsubsection{Le secteur de la chapelle orientale}

Clemens Thienpont, S. Louvion, Ebeid Mahmoud Hamid, Mohammed Ahmed Hussein, Anne-Charlotte Ybard

L'anastylose du podium romain qui portait une statue en bronze de la déesse Isis a été poursuivie. Lors de la saison 2019, la construction de la plate-forme en briques de 5,40 × $2,40 \mathrm{~m}$ qui constitue la fondation de ce podium avait été commencée. Après avoir été portée à une hauteur de $60 \mathrm{~cm}$, elle a été remplie de sable soigneusement compacté, puis couverte d'une dalle en ciment armé épaisse de $30 \mathrm{~cm}$. Cette dalle a été divisée en trois surfaces de niveaux différents pour correspondre exactement aux niveaux de fondations antiques, celui de la base, au centre, étant légèrement plus élevé que celui des deux piliers qui l'encadraient (fig. 6). 
Fig. 6. Fabrication de la dalle de fondation du podium (C. Thienpont).

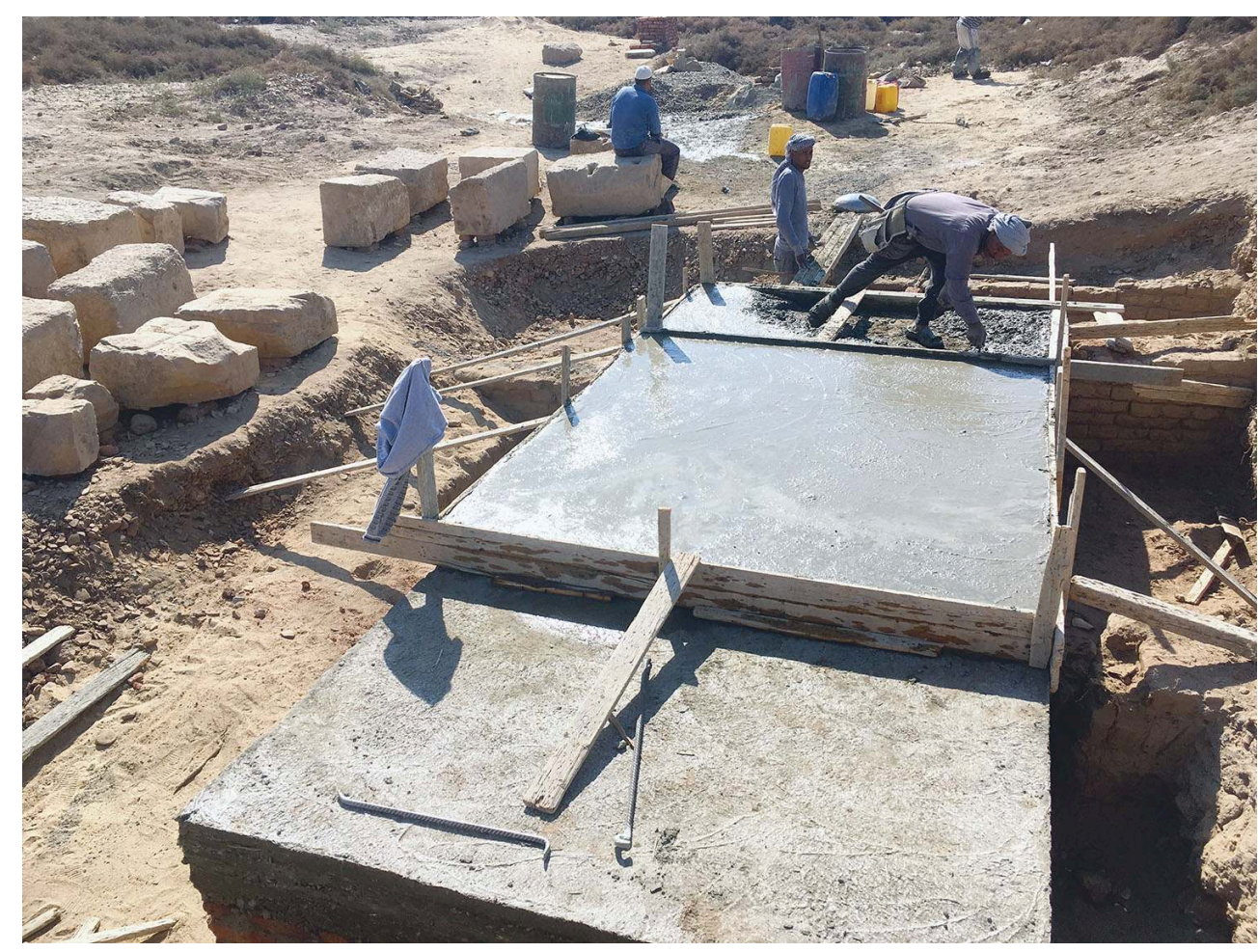

(C) Mission Coptos. 17143_2020_NDMPF_005

10 La reconstruction a pu alors être commencée, en suivant les relevés et l'étude architecturale de S. Louvion (fig. 7), et les marques d'outils visibles sur les pierres. Le podium comporte une assise de fondation en grès, surmontée de trois assises et d'une corniche en calcaire coquillier. Seules trois des faces du podium (ouest, nord et sud) étaient appareillées en pierre ; construit sur un plan en $U$, il venait s'adosser à l'est à l'épais mur de brique fermant la colonnade. Il était donc creux et devait être rempli d'un simple bourrage. Par ailleurs, l'extrémité est des assises est très irrégulière, car elle n'était que partiellement visible.

11 L'anastylose a été réalisée principalement au moyen d'un tripode et d'un palan, par une équipe coordonnée par $\mathrm{C}$. Thienpont et $\mathrm{E}$. Mahmoud. Au fur et à mesure du remontage, l'intérieur de la structure a été comblé par des éclats de pierre liés au mortier de chaux, les faces latérales et l'arrière étant complétées par des maçonneries de briques cuites hourdées au mortier. 
Fig. 7. Restitution architecturale du podium, élévation (S. Louvion).

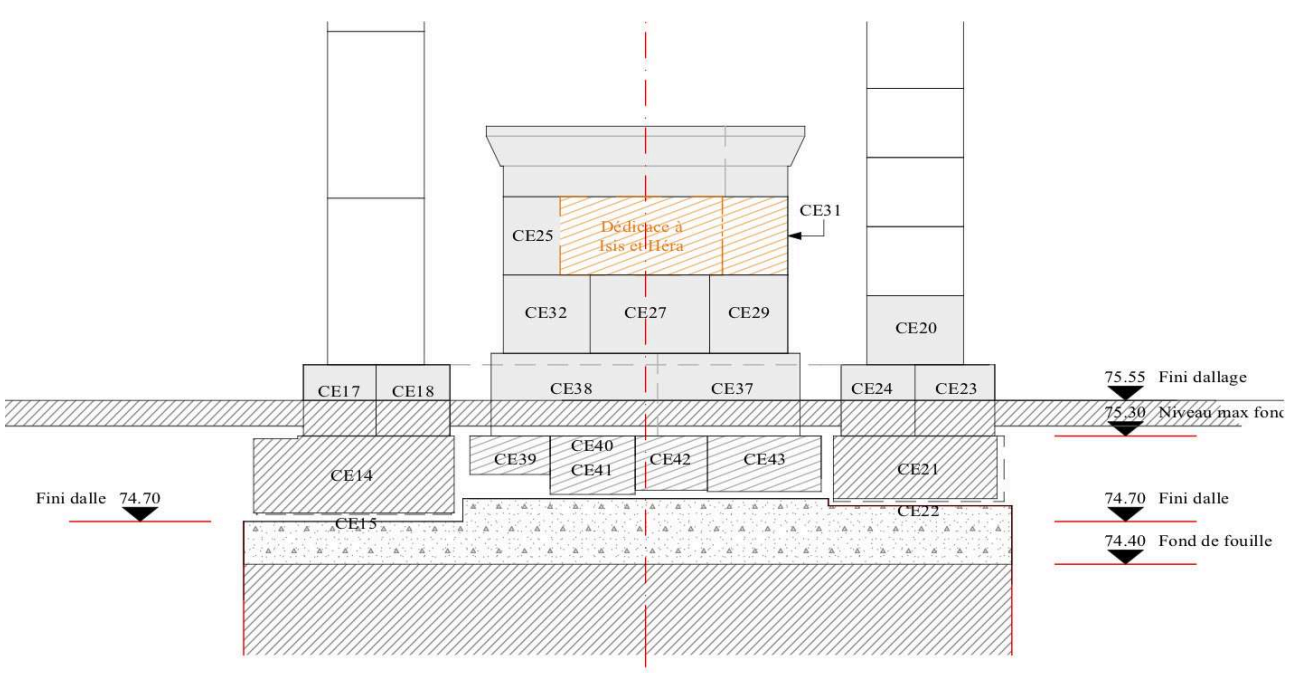

12 Légèrement plus large que les assises supérieures, la première assise forme un ressaut qui devait accentuer la monumentalité de l'ensemble. Autre élément monumental, la façade ouest de la $3^{\mathrm{e}}$ assise présentait la belle inscription dédicatoire du négociant Hermeros, gravée en 70 de n.è. Publié par G. Wagner dès 1976, ce bloc inscrit était jusqu'alors conservé dans le magasin-musée du MoTA à Qift sous le numéro d'inventaire 101. Avec l'autorisation du Comité permanent, il a pu être sorti du magasin et transporté jusqu'au site de l'anastylose par une chargeuse pour être remonté à son emplacement d'origine (fig. 8).

13 Faute de temps, la corniche sommitale n'a pas pu être remise en place cette année. Pour assurer sa protection jusqu'à la saison prochaine, le podium a été provisoirement couvert au sommet d'un lit de briques liées au mortier, et sur ses surfaces supérieures et arrière d'un enduit léger, posés par E. Mahmoud et M. Ahmed Hussein.

14 Le programme de la prochaine saison consistera à poser la corniche, remonter les bases des piliers flanquant le podium et construire à l'arrière de l'ensemble un mur de briques crues évoquant l'aspect antique du secteur.

Par ailleurs, lors du sondage mené dans ce secteur en 2019 en préalable à la reconstruction $\mathrm{du}$ podium, une épaisse couche de remblai avait livré des lots céramiques comportant un grand nombre de tessons caractéristiques du Nouvel Empire. Parmi eux, une série de plusieurs dizaines de tessons de vases peints à décor bleu a été traitée par A.-C. Ybard. Elle en a assuré le tri et l'enregistrement en photographie et en dessin, pour en permettre une étude plus approfondie. 
Fig. 8. Le podium après la mise en place du bloc portant la dédicace (C. Thienpont).

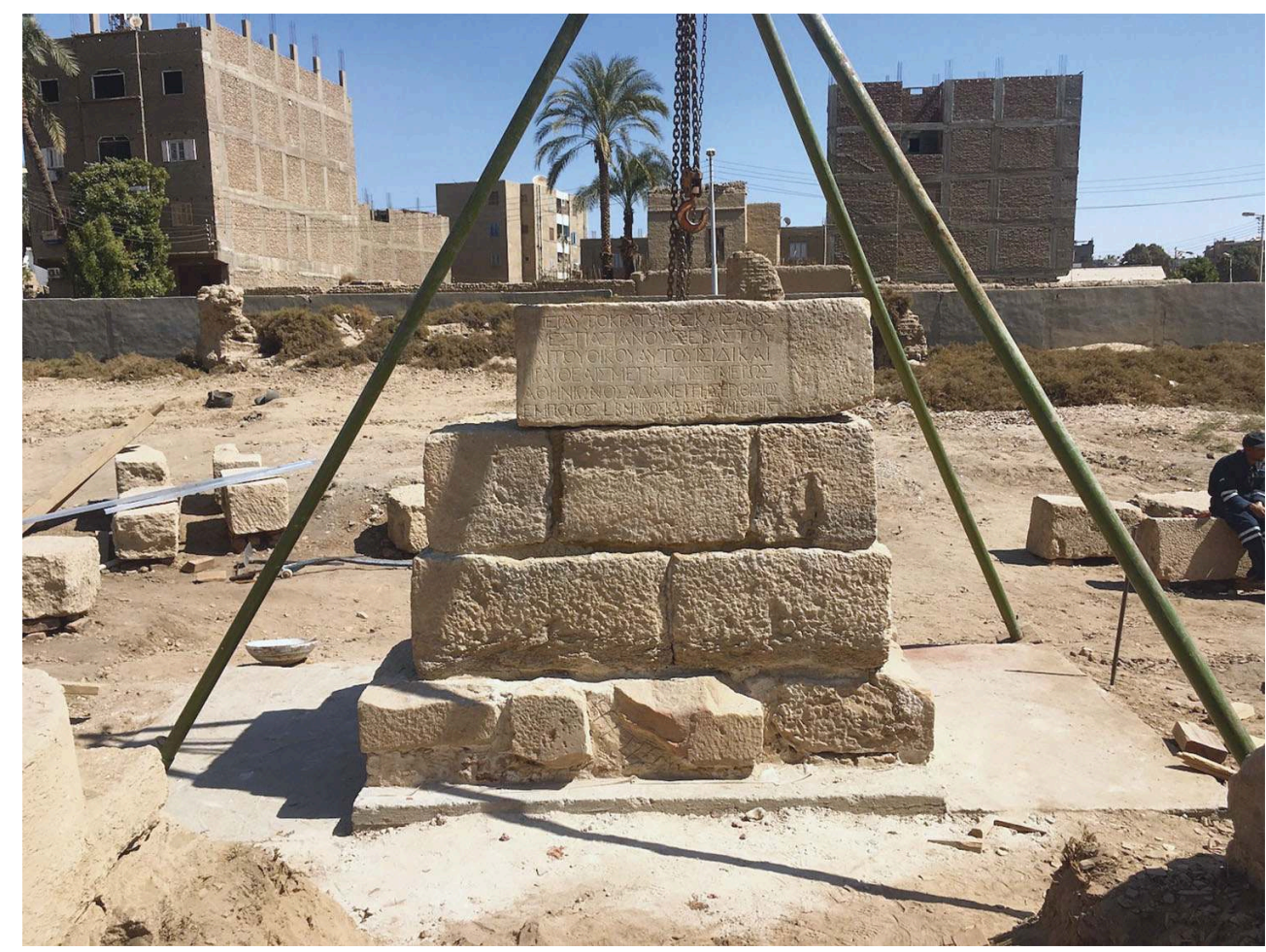

(c) Mission Coptos. 17143_2020_NDMPF_006

\subsubsection{Le mammisi de Ptolémée IV}

C. Gobeil, C. Thienpont, S. Louvion, V. Desclaux, V. Chollier, L. Pantalacci

L'objectif des travaux 2020 dans la zone du mammisi de Ptolémée IV était d'ouvrir un secteur de $10 \times 5 \mathrm{~m}$ au sud de celui déjà fouillé, à l'un des derniers endroits où le niveau de fondation, marqué par une épaisse couche de sable jaune (US 251, 252, 254, 259, 269, 271, 272, 275, 280 : voir Pantalacci 2018, p. 211-212 et fig. 2) n'avait pas encore été atteint. La surface exposée de ce secteur était formée de ce qui restait de l'épaisse couche de destruction (US 221), l'une des plus intéressantes couches fouillées autour du mammisi, ayant fourni la plus grande quantité de fragments décorés depuis le début de la fouille.

Après seulement quelques jours de travail, les ouvriers ont atteint la couche de sable jaune (US 259) déjà vue lors de la saison 2018. Comme on l'avait observé lors de cette saison passée, le sable jaune était entrecoupé de bandes de terre marron clair (US 253 et 273) caractéristiques du niveau de fondation du mammisi (fig. 9). Lors de cette opération, 176 fragments décorés ont été trouvés, dont un portant le nom d'un Ptolémée (IV ?) et un autre celui de Caligula. Ce nouveau lot de fragments devrait sans nul doute venir améliorer la compréhension du décor et de l'architecture du monument. 
Fig. 9. Le secteur du mammisi à la fin de la fouille (C. Gobeil).

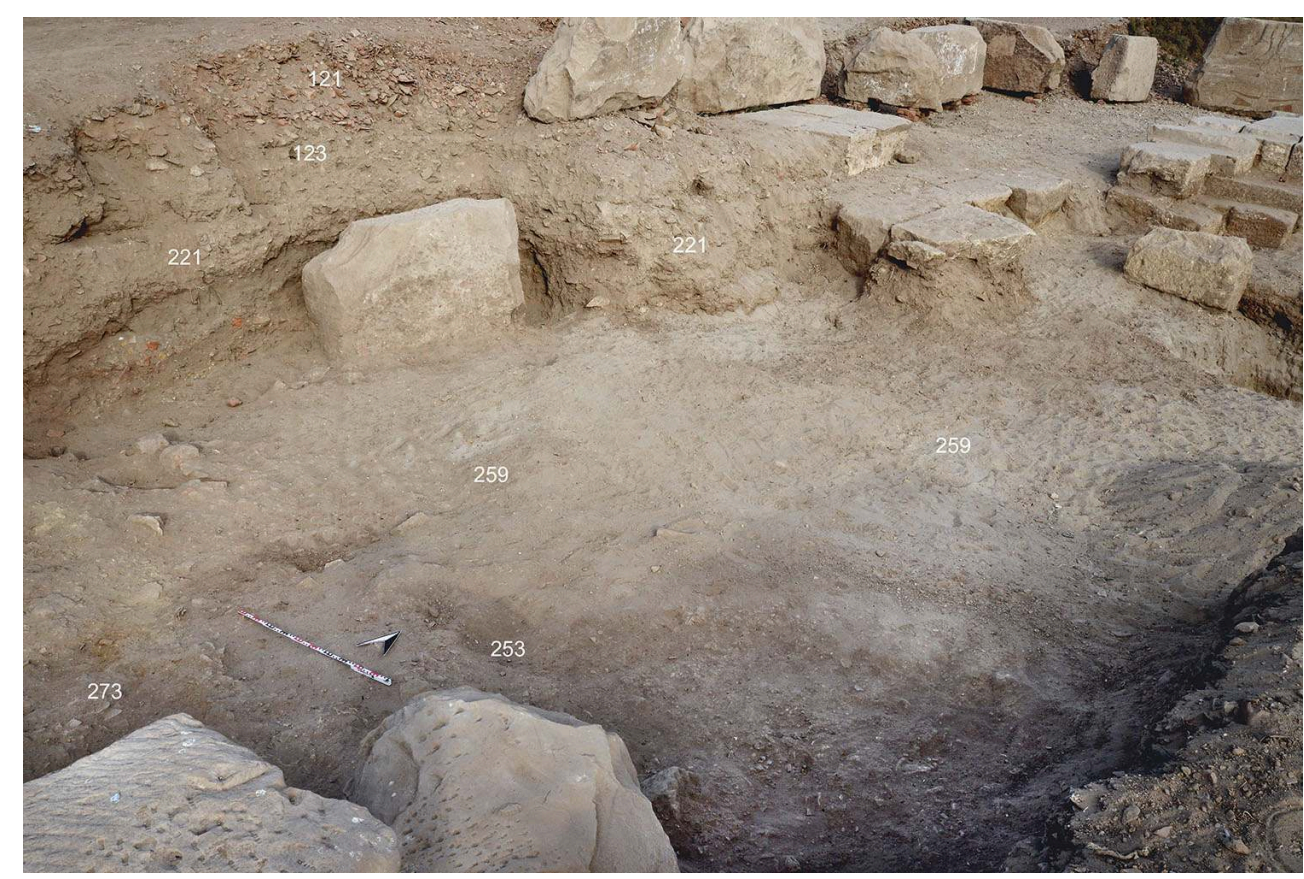

(C) Mission Coptos. 17143_2020_NDMPF_007

18 L'avancement des fouilles de cette saison a obligé l'équipe à déplacer l'une des dalles de plafond décorée (inv. M24) qui était située à l'ouest du dallage du mammisi, pour la ranger à un endroit stable et sûr. Pour ce faire, il a été nécessaire de reculer la berme ouest et d'en fouiller une petite section, exposant la suite des couches 121 et 123 vues lors des saisons précédentes. Cette opération a permis la découverte d'une nouvelle dalle de plafond en grès, située à un niveau similaire à celui du dallage du naos $(74,47 \mathrm{~m})$. Afin d'avoir une image du mammisi qui soit la plus complète possible, il faudra donc poursuivre la fouille de ce secteur l'année prochaine pour dégager cette dalle.

19 S. Louvion a poursuivi l'étude du système de couvrement du mammisi et ainsi affiné la restitution architecturale. On est maintenant certain que le naos du mammisi était précédé au sud de deux pièces distinctes, ce que le style des fragments de reliefs pariétaux préservés semble du reste confirmer.

20 L'enregistrement (inventaire, prises de mesures, dessins, photographies) des nouveaux fragments décorés trouvés cette saison a été mené par V. Desclaux et V. Chollier. Aidés de L. Pantalacci, ils ont aussi repris l'étude épigraphique et iconographique des fragments découverts lors des saisons 2017-2018. Ils sont parvenus à identifier un certain nombre de personnages et scènes appartenant au programme décoratif du mammisi et ont ainsi pu compléter certaines des scènes et motifs déjà reconnus précédemment.

\section{Formation}

B. de Faveri, MA de l'université de Bologne, et V. Marlot, doctorante à l'université Lumière - Lyon 2, ont participé durant la campagne à différents types d'activité : fouille et relevés archéologiques principalement, mais aussi documentation du matériel. 
La mission a de nouveau accueilli durant deux semaines Salim Sa'id Al-Shazly (inspecteur de Qift) et Ahmed Mohammed Aly (inspecteur de Qous), qui ont pu parfaire la formation commencée en octobre dernier, en participant ponctuellement à la fouille de la colonnade nord. Ils ont été rejoints par Magda Victor Maurice Qalini (inspecteur de Qena).

\section{Publications}

- L'article correspondant à la communication présentée en mars dernier au colloque "Mammisis of Egypt » par Laure Pantalacci et Cédric Gobeil « The Mammisi of Coptos: A Preliminary Report ", a été remis à ses éditeurs, Dagmar Budde et Ali Abd el-Halim, en mars 2020.

- La base de données Objets de Coptos hors du site, conçue par Vanessa Desclaux qui l'alimente depuis 2011, catalogue à ce jour un peu plus de 1800 objets provenant du site de Coptos, avec leurs données muséographiques et bibliographiques. Révisée une dernière fois en avril 2020, elle a été ensuite mise en ligne sur le site de la Maison de l'Orient et de la Méditerranée à Lyon, grâce à Hélène Gaboriaud-Jamet et Wei Huangfu : https://coptos.mom.fr/.

\section{Valorisation de la recherche}

Laure Pantalacci, «Coptos», in Laurent Coulon, Mélanie Cressent (éd.), Archéologie française en Égypte, BiGen 59, Le Caire, Ifao, 2019, p. 170-175.

\section{BIBLIOGRAPHIE}

PANTALACCI 2018

Laure Pantalacci, « Coptos », Rapport d'activité 2018, BIFAO-Suppl. 118, 2018, p. 210-216.

WAGNER 1976

Guy Wagner, « Une dédicace à Isis et à Héra, de la part d'un négociant d'Aden [avec 2 planches] », BIFAO 76, 1976, p. 276-281. 
INDEX

Thèmes : IFAO

Année de l'opération : 2020

anthroponymes https://ark.frantiq.fr/ark:/26678/pcrtxY8Rw2exKx, https://ark.frantiq.fr/ark:/ 26678/pcrtDRE88F0klm

lieux https://ark.frantiq.fr/ark:/26678/pcrtpMZKkpbHVW sujets https://ark.frantiq.fr/ark:/26678/pcrtb1E0Dz7cSX, https://ark.frantiq.fr/ark:/26678/ pcrtOVEVqcFc8A, https://ark.frantiq.fr/ark:/26678/pcrtjyu8fGvM12, https://ark.frantiq.fr/ark:/ 26678/pcrt5p3bwgIHaR, https://ark.frantiq.fr/ark:/26678/pcrtiwMsSdvTMw chronologie https://ark.frantiq.fr/ark:/26678/pcrtbm27waEaeg, https://ark.frantiq.fr/ark:/ 26678/pcrtaFkrKZEXbs, https://ark.frantiq.fr/ark:/26678/pcrtH5r3FYBpwe nature https://ark.frantiq.fr/ark:/26678/pcrtb1E0Dz7cSX

\section{AUTEURS}

\section{LAURE PANTALACCI}

Chef de mission, égyptologue, université Lumière Lyon 2, UMR 5189

CÉDRIC GOBEIL

Égyptologue archéologue, Museo Egizio, Turin, UMR 5189 